26

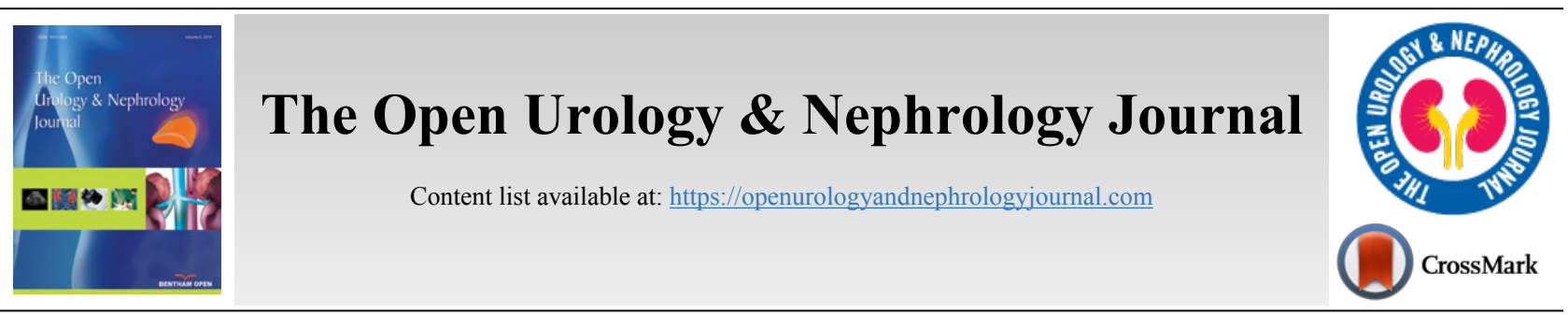

LETTER TO THE EDIT2 R

\title{
Parameters of Chronic Kidney Disease to Identify Outpatients at Increased Risk for COVID-19 Mortality: A Cohort Study of UK Biobank Participants
}

\author{
Anusua Trivedi ${ }^{1,2, *}$, W. Conrad Liles ${ }^{1,3}$, Nicholas Becker ${ }^{1,2,4}$, Catherine Egan ${ }^{5}$, Juan Lavista Ferres ${ }^{1,2}$, Aaron Lee ${ }^{1}$ and \\ Pavan K. Bhatraju $u^{1,3,6}$ \\ ${ }^{1}$ University of Washington, School of Medicine, Seattle, United States \\ ${ }^{2}$ AI for Good Research, Microsoft, United States \\ ${ }^{3}$ Department of Medicine and Sepsis Center of Research Excellence, University of Washington (SCORE-UW), Seattle, United States \\ ${ }^{4}$ University of Washington, Computer Science and Engineering, United States \\ ${ }^{5}$ Moorfields Eye Hospital NHS Foundation Trust, United Kingdom \\ ${ }^{6}$ University of Washington Division of Pulmonary, Critical Care and Sleep Medicine, Seattle, United States
}

Article History

Received: July 14, 2021

Revised: September 25, 2021

Accepted: October 21, 2021

\section{DEAR EDITOR,}

Coronavirus Disease (COVID-19) has resulted in a pandemic affecting more than a hundred countries worldwide [1]. Limited worldwide supply of vaccines against severe acute respiratory syndrome coronavirus 2 (SARS-CoV) requires policymakers to prioritize high-risk populations for inoculation. Several clinical risk factors have been suggested to increase infection risk [2]. However, it is less well-known how pre-morbid, outpatient clinical variables influence COVID-19 risk of death. We retrospectively analyzed outpatient risk factors for death in the UK Biobank (UKBB), a large-scale prospective cohort comprising over 500,000 subjects aged 40-69 years recruited in 2006-2019 [3]. In this study, subjects with recorded mortality before $31^{\text {st }}$ January $2020(\mathrm{~N}=28,930)$ were excluded since it was the date for the first recorded COVID-19 case in the UK. We performed a comprehensive study on primary care data and their associations with COVID-19 mortality in UKBB, controlling for possible confounding factors. To our knowledge, this is the broadest analysis of outpatient clinical factors and associations with COVID-19 to date.

COVID-19 outcome data were downloaded from the UKBB data portal on $23^{\text {rd }}$ February 2021. Clinical Events were obtained from the Primary Care data for COVID-19 research in UKBB. In UKBB, we set a time window of 5 years before death with COVID-19 to identify active medical diagnoses that may be risk factors with COVID-19 death. The estimated glomerular filtration rate (eGFR) was calculated by the UKBB using the Chronic Kidney Disease Epidemiology Collaboration

\footnotetext{
"Address correspondence to this author at AI for Good Research, Microsoft, United States; E-mail: antriv@microsoft.com
}

(CKD-EPI) formula, and urinary albumin to creatinine ratio (UAlb/UCr) was collected from UKBB. The diagnosis of COVID-19 was confirmed with at least one positive real-time reverse transcriptasepolymerase chain reaction (RT-PCR) test result in cases admitted with symptoms, signs, and findings (laboratory) suggestive of COVID-19. Patients without any RT-PCR positivity, and those considered as 'possible' or 'probable' cases according to the Center for Disease Control and Prevention (CDC) criteria, were not included in this study. Among 397,000 subjects in the UKBB with available GP clinical data and history of risk factors, 14,877 patients tested positive for COVID-19, and 1,994 of these patients had all the above risk factors and GP data available. We dropped most features that had more than $10 \%$ missing values resulting in 98 features. A subset of these 98 features is explained in Table 1 below.

We performed multivariable-adjusted penalized Cox proportional hazards analysis on all features, including basic demographic variables (age, sex, ethnic group), comorbidities (coronary artery disease, diabetes, hypertension, asthma, COPD, depression, dementia, history of cancer), blood measurements (e.g., blood urea and creatinine reflecting renal function), indicators of general health (number of medications taken, number of non-cancer illnesses), anthropometric measures (body mass index BMI), socioeconomic status (Townsend Deprivation index) and lifestyle risk factor (smoking and alcohol disorder status). We then completed 10fold stratified cross-validation and chose the model with the best coefficients to determine an optimal subset of features to use as predictors. Forest plots of the Cox proportional hazards regression model [4] for increased mortality in COVID-19 are shown in Fig. (1) below. Among 1,994 participants with 
COVID-19, the average age was 53.5 years with a standard deviation (SD) of 8.7 , and $1,025(51 \%)$ were male. The mortality rate was $4.1 \%$. Multivariable Cox regression results showed that older age and male gender were significantly associated with death, consistent with prior studies [5]. Compared with female participants, male participants had a significantly higher risk of death (hazard ratio (HR) 1.82, 95\% confidence interval (CI): 1.14 to $2.91, \mathrm{p}=0.013$ ). Importantly, our analysis revealed an association of baseline renal function with the risk of COVID-19-associated mortality. Improved eGFR was associated with a lower risk of death in individuals with COVID-19. Each $1 \mathrm{~mL} / \mathrm{min} / 1.73 \mathrm{~m}$ higher eGFR was associated with a $3 \%$ lower risk of death $(95 \% \mathrm{CI}$ : $1 \%$ to $5 \%$ lower, $\mathrm{p}<0.001$ ). Higher UAlb/UCr (HR $1.02,95 \%$ CI: 1.01 to $1.03, \mathrm{p}=0.001)$ was also significantly associated with death (Fig. 1).

Table 1. Subset of 98 features used in Cox Regression Model for COVID-19 survival analysis.

\begin{tabular}{|c|c|c|c|c|}
\hline S.No & Clinical Characteristics & $\begin{array}{c}\text { Overall Cohort } \\
(n=1994)\end{array}$ & $\begin{array}{l}\text { Survived } \\
(\mathrm{n}=1913)\end{array}$ & $\begin{array}{c}\text { Dead } \\
(n=81)\end{array}$ \\
\hline 1. & Age, years & $53.52+/-8.72$ & $53.08+/-8.58$ & $63.75+/-4.84$ \\
\hline 2. & Sex (Female) $(\%)$ & 49 & & \\
\hline 3. & Body mass index, $\mathrm{kg} / \mathrm{m}^{2}$ & $28.65+/-3.48$ & & \\
\hline 4. & Ethnic background - White (\%) & $\mathrm{n}=614(30.79 \%)$ & $\mathrm{n}=593(30.99 \%)$ & $\mathrm{n}=21(25.92 \%)$ \\
\hline 5. & Ethnic background - Asian (\%) & $\mathrm{n}=299(14.99 \%)$ & $\mathrm{n}=296(1547 \%)$ & $\mathrm{n}=3(3.70 \%)$ \\
\hline 6. & Ethnic background - Black (\%) & $\mathrm{n}=139(6.97 \%)$ & $n=137(7.16 \%)$ & $\mathrm{n}=2(2.46 \%)$ \\
\hline 7. & Ethnic background - Unknown (\%) & $n=942(47.24 \%)$ & $n=887(46.36 \%)$ & $\mathrm{n}=55(67.90 \%)$ \\
\hline 8. & Coronary artery disease, $\mathrm{n}(\%)$ & $\mathrm{n}=59(2.96 \%)$ & $\mathrm{n}=56(2.92 \%)$ & $\mathrm{n}=3(3.70 \%)$ \\
\hline 9. & Diabetes, n (\%) & $\mathrm{n}=159(7.97 \%)$ & $\mathrm{n}=152(7.94 \%)$ & $\mathrm{n}=7(8.64 \%)$ \\
\hline 10. & Hypertension, n (\%) & $\mathrm{n}=139(6.97 \%)$ & $\mathrm{n}=135(7.05 \%)$ & $\mathrm{n}=4(4.93 \%)$ \\
\hline 11. & Asthma, n (\%) & $\mathrm{n}=119(5.97 \%)$ & $\mathrm{n}=117(6.11 \%)$ & $\mathrm{n}=2(2.46 \%)$ \\
\hline 12. & COPD, n $(\%)$ & $\mathrm{n}=37(1.85 \%)$ & $n=35(94.59 \%)$ & $\mathrm{n}=2(2.46 \%)$ \\
\hline 13. & Depression, n (\%) & $\mathrm{n}=19(0.95 \%)$ & $\mathrm{n}=19(1.82 \%)$ & $\mathrm{n}=0(0 \%)$ \\
\hline 14. & Dementia, n (\%) & $\mathrm{n}=26(1.30 \%)$ & $\mathrm{n}=24(1.25 \%)$ & $\mathrm{n}=2(2.46 \%)$ \\
\hline 15. & History of cancer, n (\%) & $\mathrm{n}=173(8.68 \%)$ & $\mathrm{n}=168(8.78 \%)$ & $\mathrm{n}=5(6.17 \%)$ \\
\hline 16. & Systolic blood pressure, $\mathrm{mmHg}$ & $129.53+/-13.02$ & $127.49+/-12.91$ & $136.24+/-19.71$ \\
\hline 17. & Diastolic blood pressure, $\mathrm{mmHg}$ & $77.90+/-7.65$ & $77.97+/-7.51$ & $86.38+/-17.11$ \\
\hline 18. & Lymphocyte count, $10^{\wedge} 9 / \mathrm{L}$ & $2.16+/-3.09$ & $2.16+/-3.15$ & $0.91+/-1.13$ \\
\hline 19. & Platelet count, $10^{\wedge} 9 / \mathrm{L}$ & $254.68+/-46.02$ & $255.02+/-44.62$ & $215.34+/-25.65$ \\
\hline 20. & Urine albumin/creatinine ratio $\mathrm{mcg} / \mathrm{mg}$ & $3.91+/-2.85$ & $3.59+/-1.79$ & $3.73+/-4.94$ \\
\hline 21. & 21. Urine creatinine, $\mathrm{mg} / \mathrm{dL}$ & $0.86+/-0.17$ & $0.85+/-1.65$ & $0.24+/-1.78$ \\
\hline 22. & Serum blood urea nitrogen, $\mathrm{mmol} / \mathrm{L}$ & $4.93+/-1.63$ & $4.17+/-1.30$ & $4.92+/-1.64$ \\
\hline 23. & Serum $\mathrm{C}$ reactive protein level, $\mathrm{mg} / \mathrm{L}$ & $6.46+/-1.72$ & $6.43+/-5.68$ & $15.72+/-3.71$ \\
\hline 24. & $\begin{array}{r}\text { Estimated glomerular filtration rate using chronic kidney disease } \\
\text { epidemiology equation, } \mathrm{mL} / \mathrm{min} / 1.73 \mathrm{~m}\end{array}$ & $66.29+/-6.44$ & $76.47+/-5.91$ & $52.10+/-13.37$ \\
\hline
\end{tabular}

Table legend. We performed multivariable cox proportional analysis among these 98 independent features to identify variables associated with the risk of death in COVID-19.

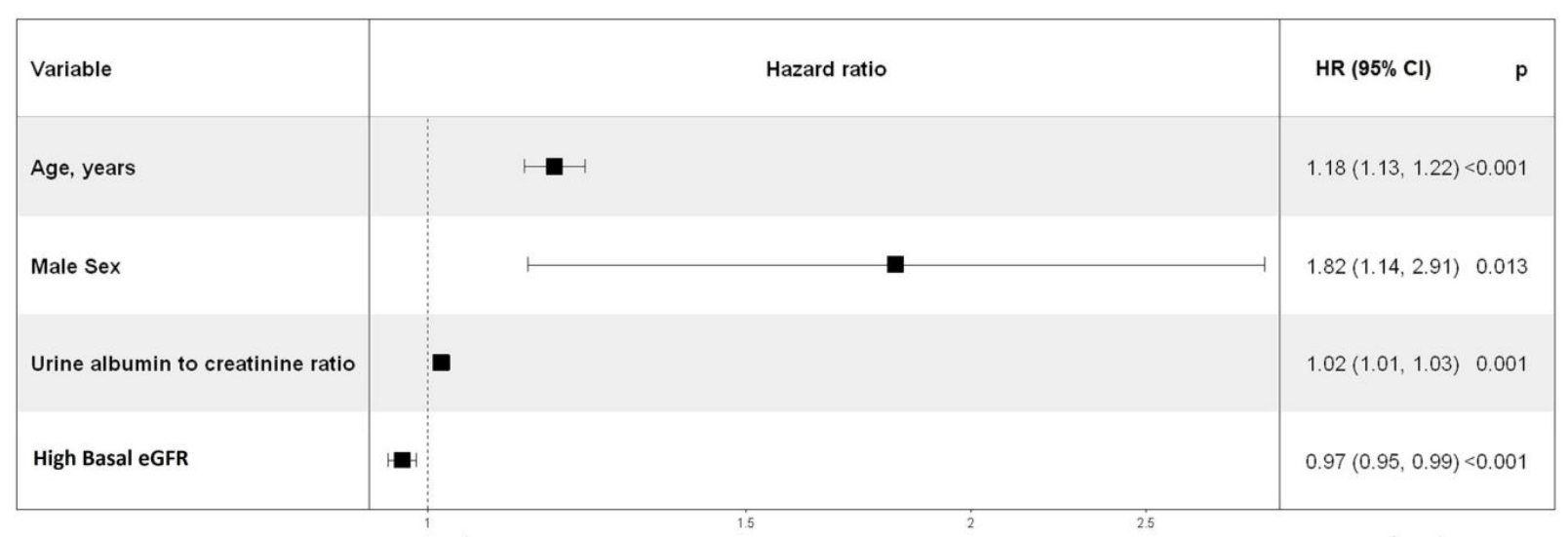

Fig. (1). Hazard Ratios for Death in COVID-19. 
COVID-19 presents an unprecedented challenge due to its complex transmission patterns and our limited understanding of risk factors associated with mortality. The acute illness may confound hospital-specific variables to understand underlying susceptibility to infection and subsequent death. This study evaluated clinical risk factors before the hospitalization that were routinely collected as part of the participant's primary care. We were able to leverage the outpatient data from the UKBB to understand underlying risk factors that could influence a patient's risk for death with COVID-19. We found statistically and clinically significant associations between mortality in COVID-19 and baseline demographics and underlying kidney function. We found that death disproportionately affected older male patients. In addition, low eGFR and high UAlb/UCr were prognostic for mortality in COVID-19. We recommend that outpatients with a history of underlying kidney issues be monitored as high-risk for COVID-19 related complications. Furthermore, our findings suggest that COVID-19 vaccine administration should be prioritized for outpatients with a history of kidney complications.

Figure legend. We performed multivariable cox proportional analysis among 98 independent features to identify variables associated with the risk of death in COVID-19. The horizontal lines represent $95 \%$ confidence intervals, with arrows indicating extensions of the intervals. Boxes represent the point estimate.

\section{CONSENT FOR PUBLICATION}

Not applicable.

\section{FUNDING}

Funding was received from the NIDDK K23DK116967 (PB), Roche Diagnostics IIS (PB, WCL), NIH/NEI K23EY029246 (AL) and a career development award from Research to Prevent Blindness (AL).

\section{CONFLICT OF INTEREST}

The authors declare no conflict of interest, financial or otherwise.

\section{ACKNOWLEDGEMENTS}

Declared none.

\section{REFERENCES}

[1] Gupta S, Hayek SS, Wang W, et al. Factors associated with death in critically ill patients with coronavirus disease 2019 in the US. JAMA Intern Med 2020; 180(11): 1436-47.

[http://dx.doi.org/10.1001/jamainternmed.2020.3596] [PMID: 32667668]

[2] Biswas M, Rahaman S, Biswas TK, Haque Z, Ibrahim B. Association of sex, age, and comorbidities with mortality in COVID-19 patients: A systematic review and meta-analysis. Intervirology 2020; 64: 1-12. [http://dx.doi.org/10.1159/000512592] [PMID: 33296901]

[3] Sudlow C, Gallacher J, Allen N, et al. UK biobank: An open access resource for identifying the causes of a wide range of complex diseases of middle and old age. PLoS Med 2015; 12(3): e1001779. [http://dx.doi.org/10.1371/journal.pmed.1001779] [PMID: 25826379]

[4] Kennedy N. Forestmodel: Forest plots from regression models. In: R Package Version. 2020; 2.

[5] Peckham H, de Gruijter NM, Raine C, et al. Male sex identified by global COVID-19 meta-analysis as a risk factor for death and ITU admission. Nat Commun 2020; 11(1): 6317.

[http://dx.doi.org/10.1038/s41467-020-19741-6] [PMID: 33298944] 